\title{
A self-similar analytical solution of spontaneous and forced imbibition in porous media
}

\author{
Xiukun Wang ${ }^{1,2 *}$, James J. Sheng ${ }^{1,2 *}$ \\ ${ }^{1}$ Unconventional Natural Gas Research Institute, China University of Petroleum, Beijing 102249, P. R. China \\ ${ }^{2}$ Bob L. Herd Department of Petroleum Engineering, Texas Tech University, Lubbock, TX, USA \\ (Received May 25, 2018; revised June 15, 2018; accepted June 16, 2018; available online June 21, 2018)
}

\section{Citation:}

Wang, X., Sheng, J.J. A self-similar analytical solution of spontaneous and forced imbibition in porous media. Advances in Geo-Energy Research, 2018, 2(3): 260-268, doi:

10.26804/ager.2018.03.04.

Corresponding author:

*E-mail: xiukun.wang@ttu.edu

*E-mail: james.sheng@ttu.edu

Keywords:

Analytical solution

spontaneous imbibition

forced imbibition

porous media

\begin{abstract}
:
Both viscous and capillary forces control the two-phase flow in porous media. The Buckley Leverett solution for viscous flow in porous media has been proposed for over a half century. While the corresponding studies of capillary dominated solutions are mainly based on the capillary tube based models. The continuum solutions are just prevail in recently years. The analytical solution of the combination of both effects is rarely investigated. A self-similar analytical solution of spontaneous and forced imbibition in porous media is proposed in this work and the corresponding concise algorithms are presented. The proposed solution successfully solves this typical non-linear partial differential equation by introducing a transformation variable and the capillary fractional flow function analog to the fractional flow function of Buckley Leverett solution. Finally, the case study is performed, which demonstrates the feasibility and accuracy of this proposed solution to a general two-phase flow condition.
\end{abstract}

\section{Introduction}

The spontaneous and forced imbibition are widely encountered in various industries including textile processing, food processing, drying engineering, petroleum engineering, etc. Imbibition in porous media refers to the process of increasing the wetting phase saturation. In this work, we focus on the water wet porous media and keep the water noted as the wetting phase. Spontaneous imbibition refers to the process of wetting phase intake spontaneously by capillary pressure. When other external forces (additional injection of the wetting phase) are added within this process, this imbibition process is called forced imbibition. For example, hydraulic fracturing in development of unconventional reservoirs (Shen et al., 2018). There are two types of spontaneous imbibition: countercurrent and cocurrent spontaneous imbibition. The former refers to that the total velocity equals to zero and that the flow directions of wetting and non-wetting phases are on the opposite. The latter refers to that the total velocity equals to the wetting phase intake velocity at the inlet and that the flow directions of wetting and non-wetting phases are the same. For forced imbibition, there is only cocurrent flow and we ignore it and only call it forced imbibition.

For spontaneous imbibition, lots of models have been proposed based on the early works by Lucas (1918) and Washburn (1921) (i.e., LW model). They used momentum balance and neglected gravity and inertial effects to derive an analytical solution for meniscus height as a function of time in a vertical capillary tube. The viscosity ratio, tortuosity, variable shaped pores and the fractal theory are further combined within this type of capillary tube based models (Handy, 1960; Li and Horne, 2000; Cai and Yu, 2011; Cai et al., 2014; Li et al., 2016; Xiao et al., 2018). However, these models still cannot consider some essential properties of porous media such as: variable wettability, the difference between countercurrent and cocurrent imbibition, etc. Apart from capillary tube based models, Schmid et al. (2011, 2013) proposed an analytical solution of spontaneous imbibition based on the continuum dynamics using capillary pressure and relative permeability curves of the porous media. This model can be understood as the capillary analog to the classical Buckley-Leverett solution (Buckley and Leverett, 1942) for viscous force dominated flow. Most affecting factors can be considered within this 
model given the corresponding capillary pressure and relative permeability curves. Recently Schmid et al. (2016) compared their calculated results with the experimental measurements of saturation profiles for a water-wet medium, which yielded some good matches. Further reviews can be found in the work by Meng et al. (2017).

For forced imbibition, the related studies are mainly numerical methods (Fayers and Sheldon, 1959; Yokoyama and Lake, 1981). Chen et al. (1990) and McWhorter and Sunada (1990) did some primary works for the corresponding analytical solutions. Actually the spontaneous imbibition model by Schmid et al. $(2011,2013)$ is also derived from McWhorter and Sunada (1990)'s work. Analog to the fractional flow function, they introduced the concept of capillary fractional flow function defined as the ratio of wetting phase flow rate for various wetting phase saturation to that at inlet with the highest wetting phase saturation as shown in Eq. (6). Their derived solution of forced imbibition is only applicable to piston-like displacement as noted by Chen et al. (1990) and the analytical solution involves the implicit integration, which costs thousands of iterations before the convergence. Recently, Bjørnarå and Mathias (2013) improved the solution using a pseudospectral Chebyshev differentiation matrix. Schmid et al. (2011, 2013, 2016) only focused on spontaneous imbibition and the forced imbibition was not presented in their works.

In this work, we propose a self-similar analytical solution of spontaneous and forced imbibition by introducing the capillary fractional flow function (Chen et al., 1990; McWhorter and Sunada, 1990; Schmid et al., 2016). Corresponding concise algorithms for solving the analytical solution are also proposed using the combination of Runge-Kutta and Bisection methods. Finally, a case study is conducted to validate our solution and algorithm.

\section{A general analytical solution of spontaneous and forced imbibition}

The incompressible linear oil-water two-phase flow in porous media is considered in this work. Water is set as the wetting phase and oil as the non-wetting phase. Based on water phase mass conservation, the following equation is obtained as (Chen et al., 1990):

$$
\phi \frac{\partial S_{w}}{\partial t}+\frac{\partial u_{w}}{\partial x}=0
$$

where $u_{w}, S_{w}$ and $\phi$ are the Darcy velocity for the water phase, water phase saturation and the porosity of a porous medium, respectively.

Ignoring the gravity effect, the water phase Darcy flow velocity is expressed by (Dake, 1978):

$$
u_{w}=\frac{1}{1+\frac{k_{r o}\left(S_{w}\right) \mu_{w}}{k_{r w}\left(S_{w}\right) \mu_{o}}}\left(u_{t}+k \frac{k_{r o}\left(S_{w}\right)}{\mu_{o}} \frac{\partial p_{c}}{\partial x}\right)
$$

where $\mu, k, k_{r}$ and $p_{c}$ are the fluid viscosity, absolute permeability, relative permeability and capillary pressure, respectively; Subscript $o, w$ and $t$ refer to the oil phase, the water phase and the total two-phase, respectively.
Conventionally, the factional flow function is defined as (Buckley and Leverett, 1942; Dake, 1978):

$$
f_{w}\left(S_{w}\right)=\frac{1}{1+\frac{k_{r o}\left(S_{w}\right) \mu_{w}}{k_{r w}\left(S_{w}\right) \mu_{o}}}
$$

Defining $D\left(S_{w}\right)=-k \frac{k_{r o}\left(S_{w}\right) k_{r w}\left(S_{w}\right)}{\mu_{o} k_{r w}\left(S_{w}\right)+\mu_{w} k_{r o}\left(S_{w}\right)} \frac{\partial p_{c}}{\partial S_{w}}$, the Eq. (2) is expressed in a concise form in Eq. (4).

$$
u_{w}=u_{t} f_{w}\left(S_{w}\right)-D\left(S_{w}\right) \frac{\partial S_{w}}{\partial x}
$$

The mathematical problem is to solve Eqs. (1) and (4) with the following initial and boundary conditions in Eq. (5).

$$
\begin{array}{r}
S_{w}(x, t=0)=S_{w c} \\
S_{w}(x=\infty, t>0)=S_{w c} \\
S_{w}(x=0, t>0)=S_{w}^{*}
\end{array}
$$

where $S_{w c}$ and $S_{w}^{*}$ are the water phase saturation for the initial condition (connate water saturation) and at the inlet, respectively. Note that the inlet water saturation $S_{w}^{*}$ is the maximum saturation, which equals to $1-S_{o r}$ for water wet porous media, where the $S_{o r}$ is the residual oil saturation.

Analog to the Buckley and Leverett theory, a capillary fractional flow function $F\left(S_{w}\right)$ is introduced (Chen et al., 1990; McWhorter and Sunada, 1990; Schmid et al., 2011) as:

$$
u_{w}=u_{w}^{0} F\left(S_{w}\right)
$$

where $u_{w}^{0}$ is the water injection velocity at the inlet, which implies that $F\left(S_{w}^{*}\right)=1$.

Substituting Eq. (6) into Eq. (1), the following formula is obtained after introducing a transformation variable $\omega=$ $x / \sqrt{t}$.

$$
\phi \frac{d S_{w}}{d \omega}\left(-\frac{\omega}{2 t}\right)+u_{w}^{0} \frac{d F}{d S_{w}} \frac{d S_{w}}{d \omega}\left(\frac{1}{\sqrt{t}}\right)=0
$$

To simplify Eq. (7), the water injection velocity at the inlet is set as $u_{w}^{0}=A / \sqrt{t}$, where $A$ is a constant parameter. We can get:

$$
\phi \frac{d S_{w}}{d \omega} \frac{\omega}{2}=A \frac{d F}{d S_{w}} \frac{d S_{w}}{d \omega}
$$

Then

$$
\omega=\frac{2 A}{\phi} \frac{d F}{d S_{w}}=\frac{2 A}{\phi} F^{\prime}
$$

Then

$$
\frac{d S_{w}}{d \omega}=\frac{\phi}{2 A F^{\prime \prime}}
$$

Substituting Eq. (10) into Eq. (4), the following equation is obtained:

$$
u_{w}=u_{t} f_{w}-D \frac{\phi}{2 A F^{\prime \prime}} \frac{1}{\sqrt{t}}
$$




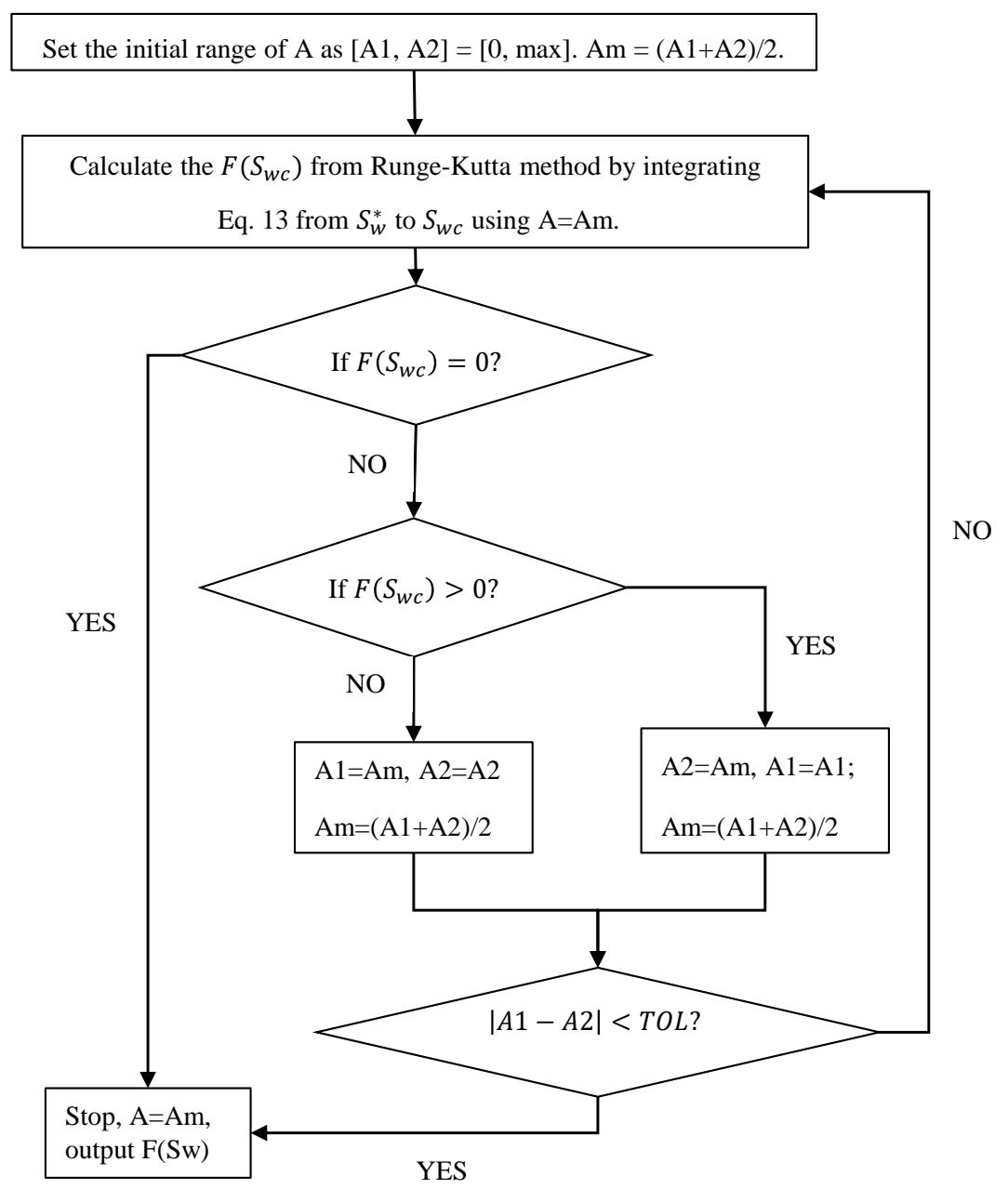

Fig. 1. Flow chart of the algorithm for spontaneous imbibition calculation: Scenario \#1 \& \#2.

The combination of Eqs. (6) and (11) yields:

$$
\frac{A}{\sqrt{t}} F=u_{t} f_{w}-D \frac{\phi}{2 A F^{\prime \prime}} \frac{1}{\sqrt{t}}
$$

The total velocity is set as $u_{t}=B / \sqrt{t}$ ( $B$ is another constant parameter) in order to eliminate the variable $t$. Finally, the self-similar analytical solution of spontaneous and forced imbibition is formulated as:

$$
\left(A F-B f_{w}\right) F^{\prime \prime}=-\frac{D \phi}{2 A}
$$

Once the capillary fractional flow function $F\left(S_{w}\right)$ is calculated from Eq. (13), the corresponding water saturation propagation profile is obtained from Eq. (9).

Three scenarios exist in this proposed general solution: \#1 Countercurrent spontaneous imbibition; \#2 Cocurrent spontaneous imbibition; and \#3 Cocurrent forced imbibition. For the first scenario, the total flow velocity $u_{t}=0$, i.e., $B=0$ and $A$ is unknown; For the second scenario, the total flow velocity equals to the injection velocity at the inlet, i,e., $u_{t}=$ $u_{w}^{0}$, therefore, $A=B$ which is unknown. For both Scenario \#1 $\& \# 2$, the mathematical problem is to solve Eq. (13) with the boundary conditions from Eq. (5) and an unknown $A$, however, it is not solvable until the additional boundary condition $\left(F^{\prime}\left(S_{w}\right)^{*}=0\right)$ is added to make sure that the spontaneous imbibition velocity is zero given $S_{w}=S_{w}^{*}$. For Scenario \#3, the total flow velocity equals to the injection velocity at the inlet, i.e., $u_{t}=u_{w}^{0}$. Therefore, $A=B$, however, it is a given value unlike the second scenario. The mathematical problem is to solve Eq. (13) with the boundary conditions from Eq. (5) and a given $A$ and no additional boundary condition is needed. Note that the value of $A$ is larger than that in Scenario \#2 to make sure the forced imbibition happens. All the above statements are summarized in Table 1.

\section{Algorithms}

\subsection{Algorithm for spontaneous imbibition}

For Scenario \#1 \& \#2, the algorithm is identical except for the different forms of Eq. (13) as shown in Table 1. We propose a new algorithm based on Runge-Kutta and Bisection methods (Chapra and Canale, 1988). Since the value of $A$ is within a range of 0 to a positive maximum. Initially we present a large enough range for $A$, then use Runge-Kutta method to get $F\left(S_{w c}\right)$ by integrating Eq. (13) from $S_{w}^{*}$ to $S_{w c}$ assigning 
Table 1. Three scenarios for the self-similar analytical solution from Eq. (13).

\begin{tabular}{llll}
\hline Scenarios & Simplified forms of Eq. (13) & To be solved & Additional conditions besides Eq. (5) \\
\hline \#1 Countercurrent spontaneous imbibition & $F F^{\prime \prime}=-\frac{D \phi}{2 A^{2}}$ & $F\left(S_{w}\right), A$ & $F^{\prime}\left(S_{w}^{*}\right)=0$ \\
\#2 Cocurrent spontaneous imbibition & $\left(F-f_{w}\right) F^{\prime \prime}=-\frac{D \phi}{2 A^{2}}$ & $F\left(S_{w}\right), A$ & $F^{\prime}\left(S_{w}^{*}\right)=0$ \\
\#3 Forced imbibition & $\left(F-f_{w}\right) F^{\prime \prime}=-\frac{D \phi}{2 A^{2}}$ & $F\left(S_{w}\right)$ & $A$ \\
\hline
\end{tabular}

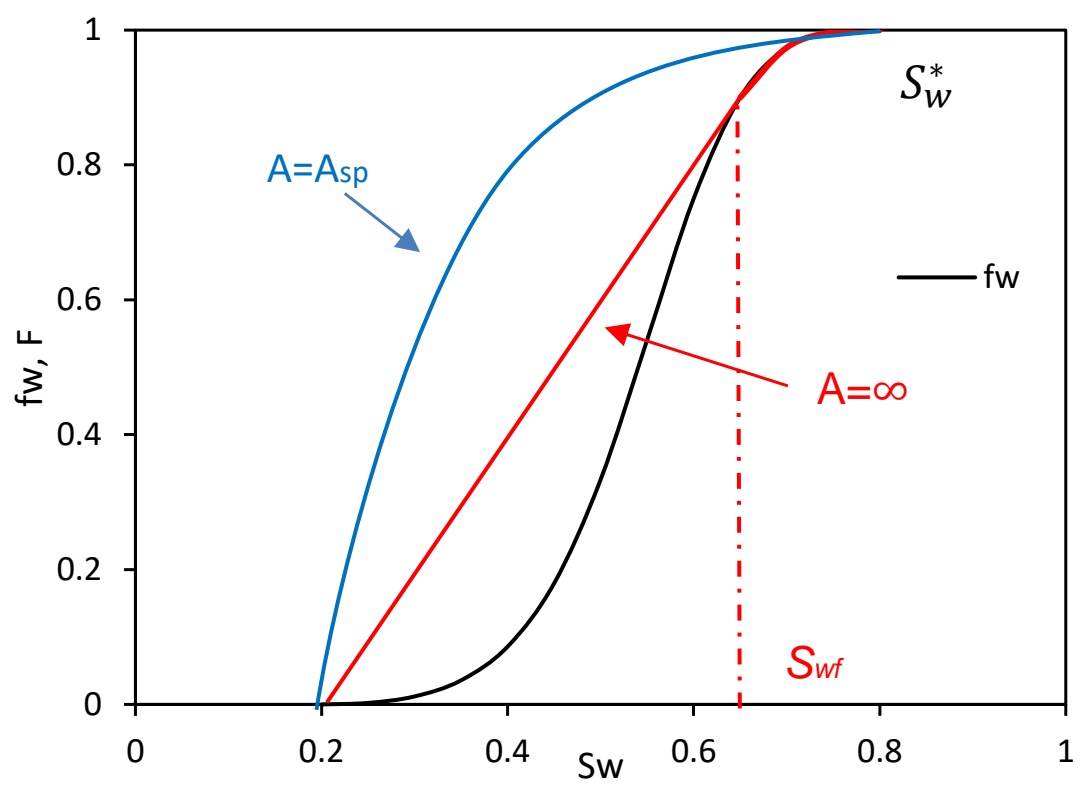

Fig. 2. Schematic of $F\left(S_{w}\right)$ with respect to different values of $A$.

$F^{\prime}\left(S_{w}^{*}\right)=0$. If $F\left(S_{w}\right)=0$, then the value of $A$ is what we want to get, otherwise, using the Bisection method to shorten the possible range of $A$. The details of this algorithm is shown in Fig. 1 . In the end, $A$ and $F\left(S_{w}\right)$ will be calculated and the corresponding water imbibition profile and oil recovery factor will be obtained. The numerical Runge-Kutta integral of Eq. (13) can be implemented using the "ode45" function in the Matlab environment (Shampine and Reichelt, 1997).

\subsection{Algorithm for forced imbibition}

For Scenario \#3: forced imbibition, the value of $A$ is given, which is larger than that $A_{s p}$ obtained from cocurrent spontaneous imbibition since additional water is injected. Therefore, $F\left(S_{w}\right)$ should always be no less than $f_{w}\left(S_{w}\right)$ as noted by Chen et al. (1990). A schematic of $F\left(S_{w}\right)$ with respect to different values of $A$ is shown in Fig. 2. When $f_{w}^{\prime}\left(S_{w}^{*}\right)=0, A$ $=A_{s p}$ which corresponds to cocurrent spontaneous imbibition as shown in the blue curve. When $A=\infty, F\left(S_{w}\right)$ becomes the red curve, which consists of two parts: the linear part ( $S_{w c}$ to $\left.S_{w f}\right)$ and the $f_{w}\left(S_{w}\right)$ part $\left(S_{w f}\right.$ to $\left.S_{w}^{*}\right)$. $S_{w f}$ refers to the frontal water saturation obtained from Buckley Leverett theory (Dake, 1978). When $A=\infty$, the flow is dominated by the viscous forces and the capillary effect becomes negligible, which demonstrates the compatibility of our proposed analytical model. Given $A>A_{s p}$, the corresponding $F\left(S_{w}\right)$ function is between these two curves as shown in Fig. 2. Therfore, firstly we need to check the value of $f_{w}^{\prime}\left(S_{w}^{*}\right)$. If $f_{w}^{\prime}\left(S_{w}^{*}\right)>0$, the initial guess for $F^{\prime}\left(S_{w}^{*}\right)$ is $f_{w}^{\prime}\left(S_{w}^{*}\right)$. Given a value of $A$, we use RungeKutta method to get $F\left(S_{w c}\right)$ by integrating Eq.(13) from $S_{w}^{*}$ to $S_{w c}$. If $F\left(S_{w c}\right)>0$, the Bisection method is used to decrease the guess for $F^{\prime}\left(S_{w}^{*}\right)$ as $\frac{f_{w}^{\prime}\left(S_{w}^{*}\right)+0}{2}$ and do the iteration to get $F\left(S_{w}\right)=0$; If $F\left(S_{w c}\right)<0$, the Bisection method is used in another way by changing the integral range from $S_{w}^{*}$ to $S_{w c}$ to $\frac{S_{w}^{*}+S_{w f}}{2}$ to $S_{w c}$. Still the Runge-Kutta method is used to get $F\left(S_{w c}\right)$ assigning $F^{\prime}\left(\frac{S_{w}^{*}+S_{w f}}{2}\right)=f_{w}^{\prime}\left(\frac{S_{w}^{*}+S_{w f}}{2}\right)$. The process is also iterated until $F\left(S_{w c}\right)=0$. If initially $f_{w}^{\prime}\left(S_{w}^{*}\right)=0$ (actually this situation is more common and general), the algorithm is identical to the above by shortening the integral range from $S_{w}^{*}$ to $S_{w c}$ to $\frac{S_{w}^{*}+S_{w f}}{2}$ to $S_{w c}$ and do the iteration to get $F\left(S_{w c}\right)$ $=0$ and the detail of the corresponding algorithm is shown in Fig. 3. Finally, the solution of $F\left(S_{w}\right)$ and the corresponding water imbibition profile are calculated.

\section{Case study}

Before proceeding the calculation, the capillary pressure is expressed in a dimensionless from by:

$$
p_{c}\left(S_{w}\right)=p_{c}^{\max } J\left(S_{w}\right)=p_{c}^{\max }\left(\frac{S_{w}^{*}-S_{w}}{S_{w}^{*}-S_{w c}}\right)^{a}
$$




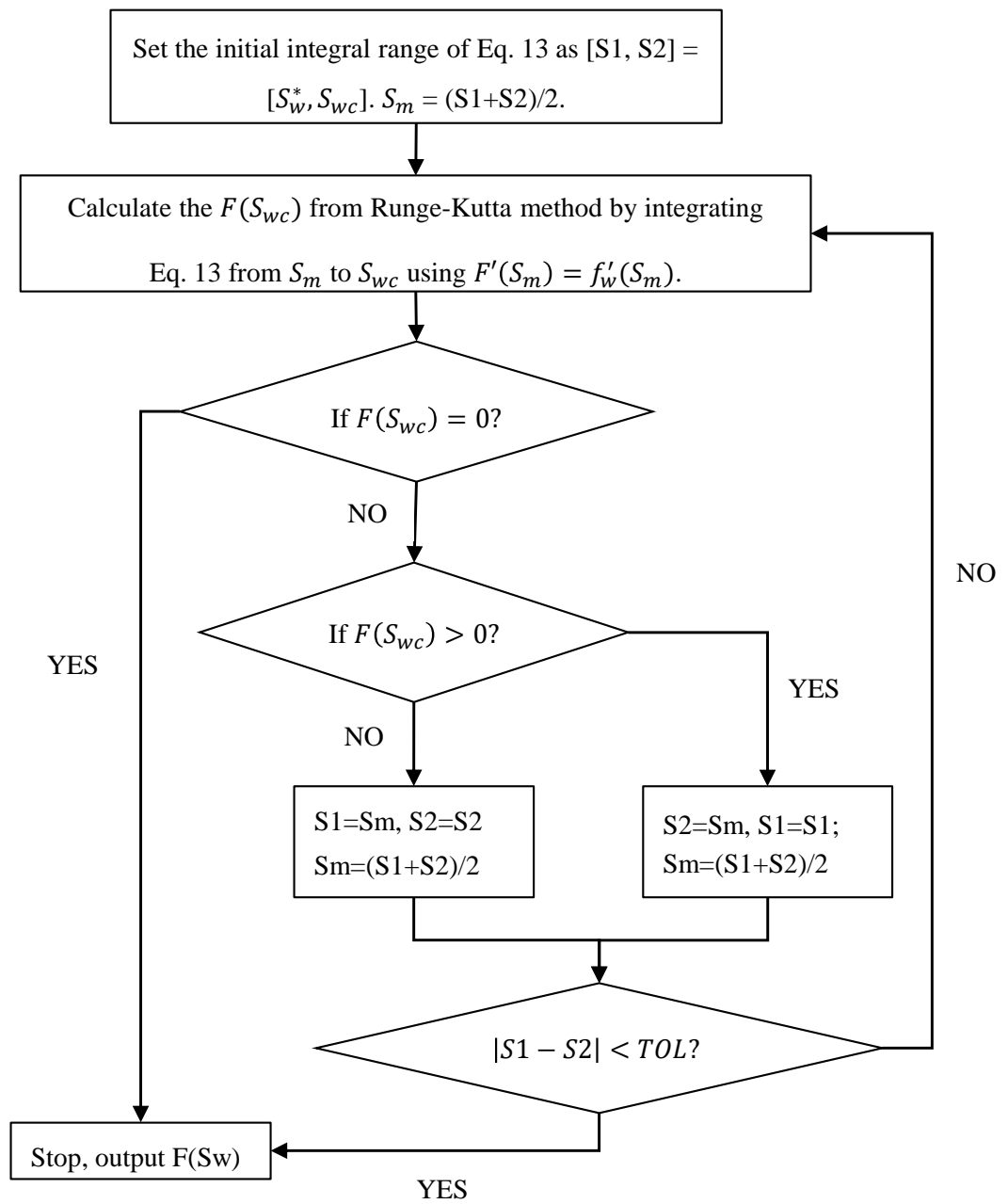

Fig. 3. Flow chart of the algorithm for forced imbibition.

Table 2. Parameters for case studies.

\begin{tabular}{llllllllllll}
\hline$p_{c}^{\max }(\mathrm{kPa})$ & $k(\mathrm{D})$ & $\mu_{o}(\mathrm{cP})$ & $\mu_{w}(\mathrm{cP})$ & $\phi$ & $a$ & $b_{1}$ & $b_{2}$ & $c_{1}$ & $c_{2}$ & $S_{w c}$ & $S_{w}^{*}$ \\
\hline 30 & 1 & 1 & 1 & 0.25 & 4 & 0.8 & 0.2 & 3 & 2 & 0.2 & 0.8 \\
\hline
\end{tabular}

where $p_{c}^{\max }$ is the maximum capillary pressure; $a$ and $J\left(S_{w}\right)$ are a positive parameter and the dimensionless capillary pressure function, respectively.

Recall the definition of $D\left(S_{w}\right)$, we can get the flowing expression from Eq. (14).

$$
D\left(S_{w}\right)=k \frac{k_{r o}\left(S_{w}\right) k_{r w}\left(S_{w}\right)}{\mu_{o} k_{r w}\left(S_{w}\right)+\mu_{w} k_{r o}\left(S_{w}\right)} \frac{a p_{c}^{\max }\left(S_{w}^{*}-S_{w}\right)^{a-1}}{\left(S_{w}^{*}-S_{w c}\right)^{a}}
$$

The Brook-Corey's correlations (Peters, 2012) for relative permeability curves are used as shown in Eq. (16):

$$
\begin{gathered}
k_{r o}=b_{1}\left(1-\frac{S_{w}-S_{w c}}{S_{w}^{*}-S_{w c}}\right)^{c_{1}} \\
k_{r w}=b_{2}\left(\frac{S_{w}-S_{w c}}{S_{w}^{*}-S_{w c}}\right)^{c_{2}}
\end{gathered}
$$

where $b_{1}, b_{2}, c_{1}$ and $c_{2}$ are all positive parameters.
The parameters used in the case study is presented in Table 2 and corresponding dimensionless pressure and relative permeability curves are shown in Figs. 4 and 5.

Firstly, the spontaneous countercurrent and cocurrent imbibition are calculated using the algorithm from Fig. 1. The calculated $F\left(S_{w}\right)$ functions are presented along with $f_{w}\left(S_{w}\right)$ in Fig. 6 and the water spontaneous imbibition profiles are shown in Fig. 7 at the scale of the transformation variable $\omega$ $=x / \sqrt{t}$. According to the figures, the $F\left(S_{w}\right)$ of countercurrent spontaneous imbibition is slightly higher than that of cocurrent spontaneous imbibition. Since the velocity of a certain saturation is proportional to the derivative of $F\left(S_{w}\right)$ at the axis of $\omega=x / \sqrt{t}$, the saturation profile of cocurrent spontaneous imbibition moves slightly faster than that of countercurrent spontaneous imbibition as shown in Fig. 7. The larger the water saturation, the faster does the profile propagate. For $S_{w}$ $=S_{w}^{*}$, the profile doesn't move. Besides, the calculated As for 


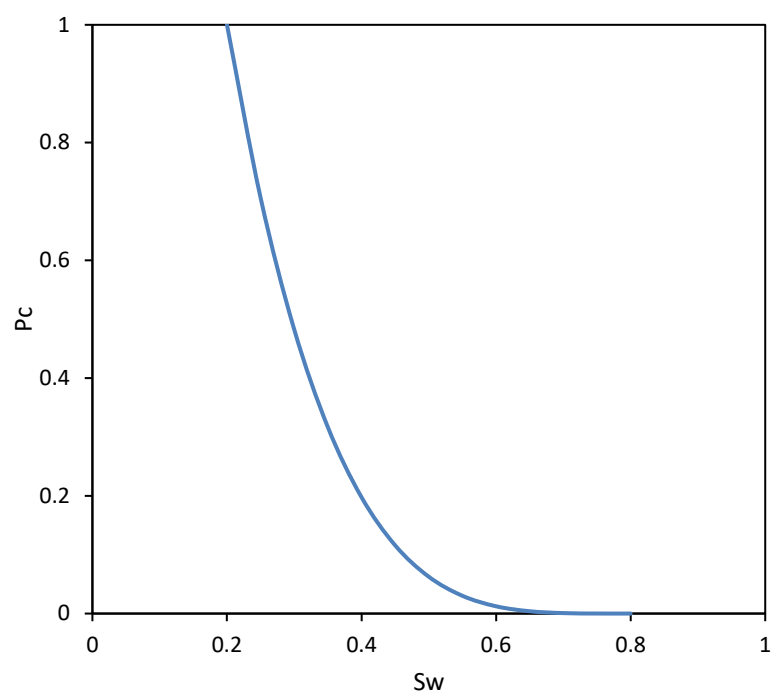

Fig. 4. Dimensionless capillary pressure vs. water saturation.

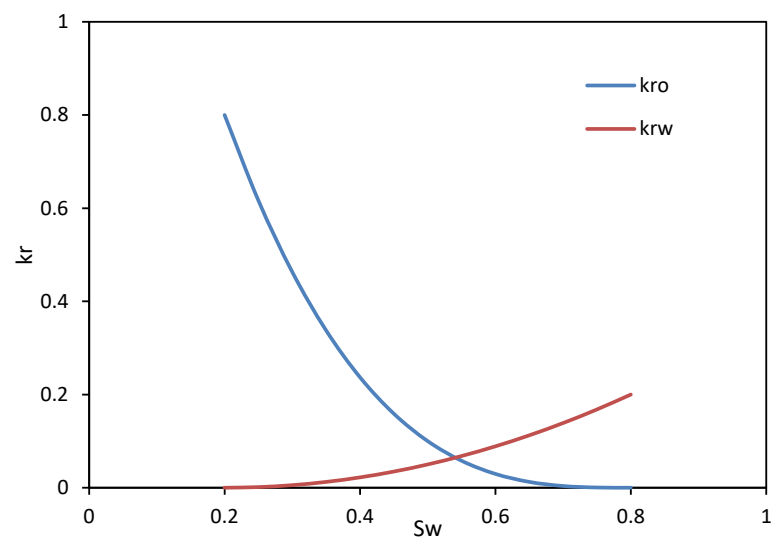

Fig. 5. Relative permeability vs. water saturation.

countercurrent and concurrent imbibition are $9.67 \times 10^{-5}$ $\mathrm{m} / \sqrt{\mathrm{s}}$ and $1.27 \times 10^{-4} \mathrm{~m} / \sqrt{\mathrm{s}}$ and the recovery factors can be calculated using $R F=\int_{0}^{t} \frac{u_{t}}{\phi} d t=\int_{0}^{t} \frac{A}{\phi \sqrt{t}} d t=\frac{2 A \sqrt{t}}{\phi}$ assuming a unit characteristic length, as shown in Fig. 8. The cocurrent spontaneous imbibition yields a higher recovery given the same time.

Secondly, the forced imbibition process is calculated using the parameters from Table 2 and the algorithm from Fig. 3. Given different injection velocities (different values of $A$ ), the corresponding capillary fractional flow function $F\left(S_{w}\right)$ are presented in Fig. 9. $A_{s p}$ refers to the condition of cocurrent spontaneous imbibition. And the conditions of 2 and 5 times of the $A_{s p}$ are used to study the forced imbibition process. The propagation profiles of water saturation are shown in Fig. 10 and the corresponding Buckley Leverett solutions (Dake, 1978; Peters, 2012) are also presented, which ignore the capillary pressure between the oil and water phases. According to the figures, the curvature of $F\left(S_{w}\right)$ decreases when the injection velocity increases ( $A$ increases). The $F\left(S_{w}\right)$ consists of two parts: one is identical to a part of $f_{w}\left(S_{w}\right)$; the other is a less tortuous curve connecting the transition point from the first

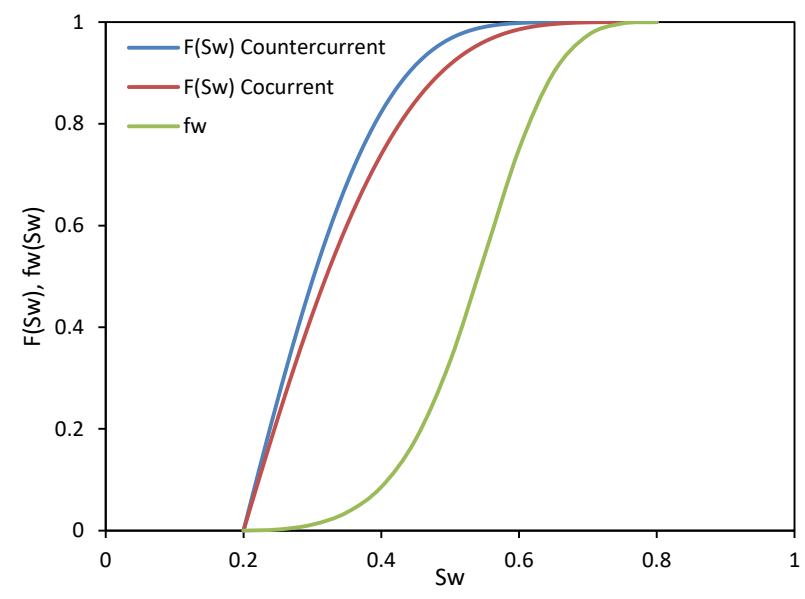

Fig. 6. Calculated $F\left(S_{w}\right)$ of countercurrent and cocurrent spontaneous imbibition for the parameters given in Table 2 .

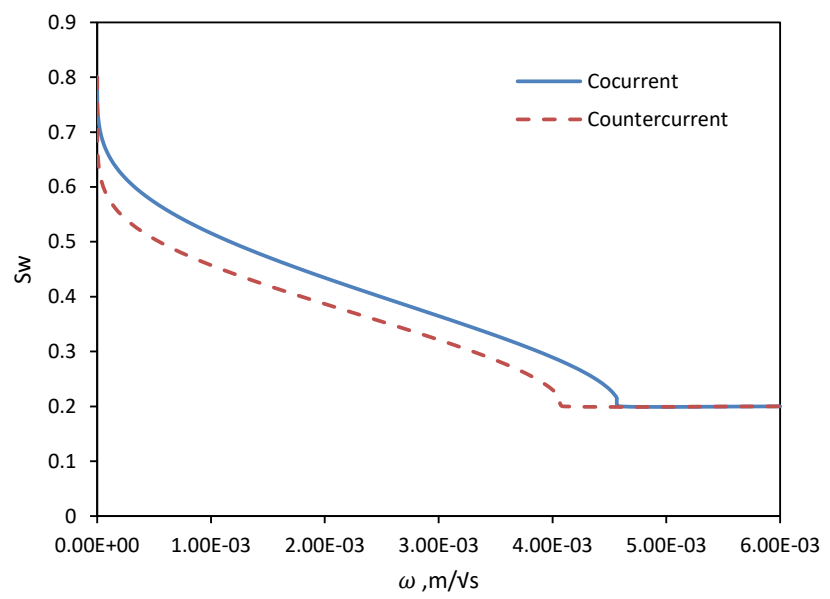

Fig. 7. Spontaneous imbibition profiles $S_{w}$ vs. $\omega=x / \sqrt{t}$.

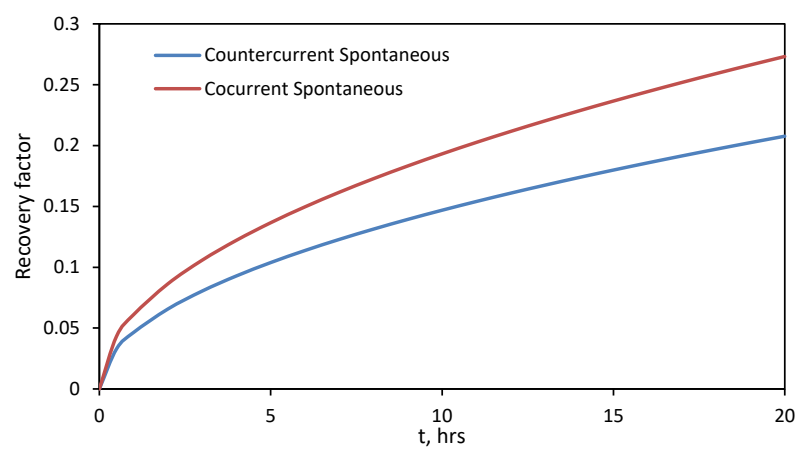

Fig. 8. Recovery factor of spontaneous imbibition.

part to the point of $\left(S_{w c}, 0\right)$. When $A=5 A_{s p}$, the second part become quite close to a straight line and the connecting saturation is approaching water frontal saturation $S_{w f}$. The saturation propagation profiles present a more practical point of views as shown in Fig. 10. When the injection velocity (value of $A$ ) increases, the saturation propagates faster for the scale of $\omega=$ 


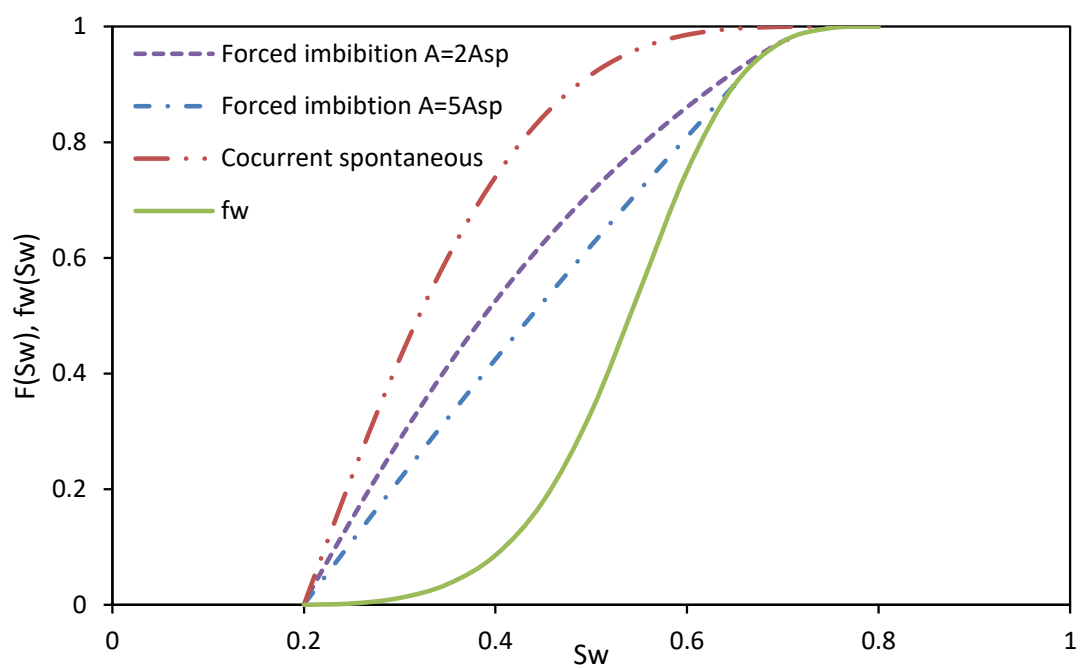

Fig. 9. Capillary fractional flow function $F\left(S_{w}\right)$ of forced imbibition for different injection velocities (different values of $A$ ).

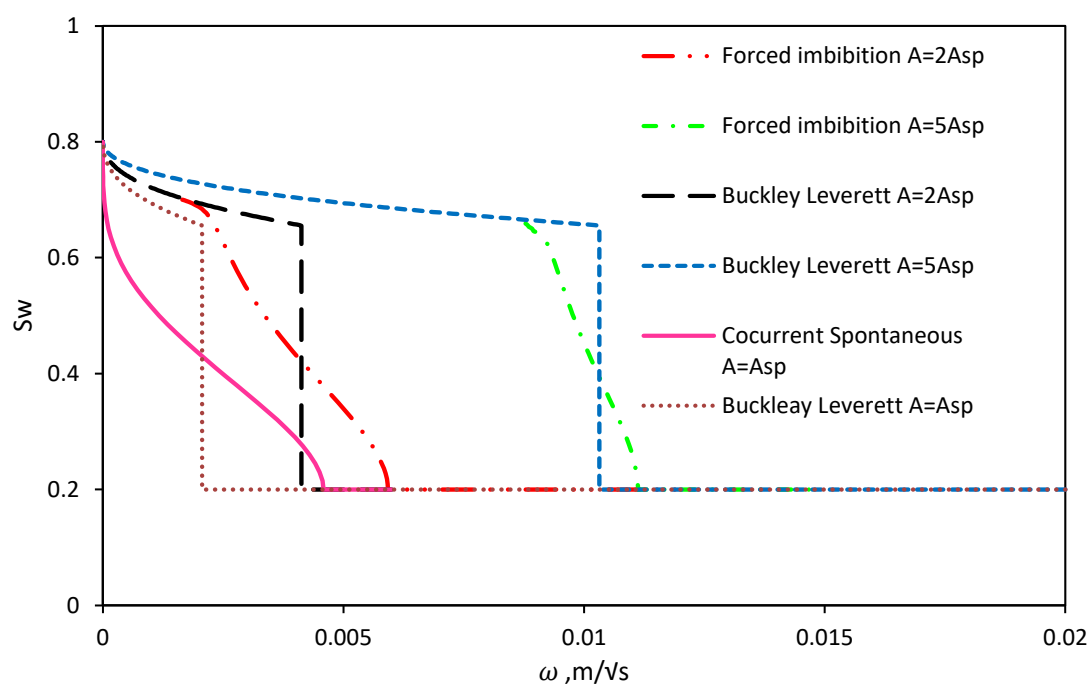

Fig. 10. Saturation propagation profiles of forced imbibition for different injection velocities (different values of $A$ ).

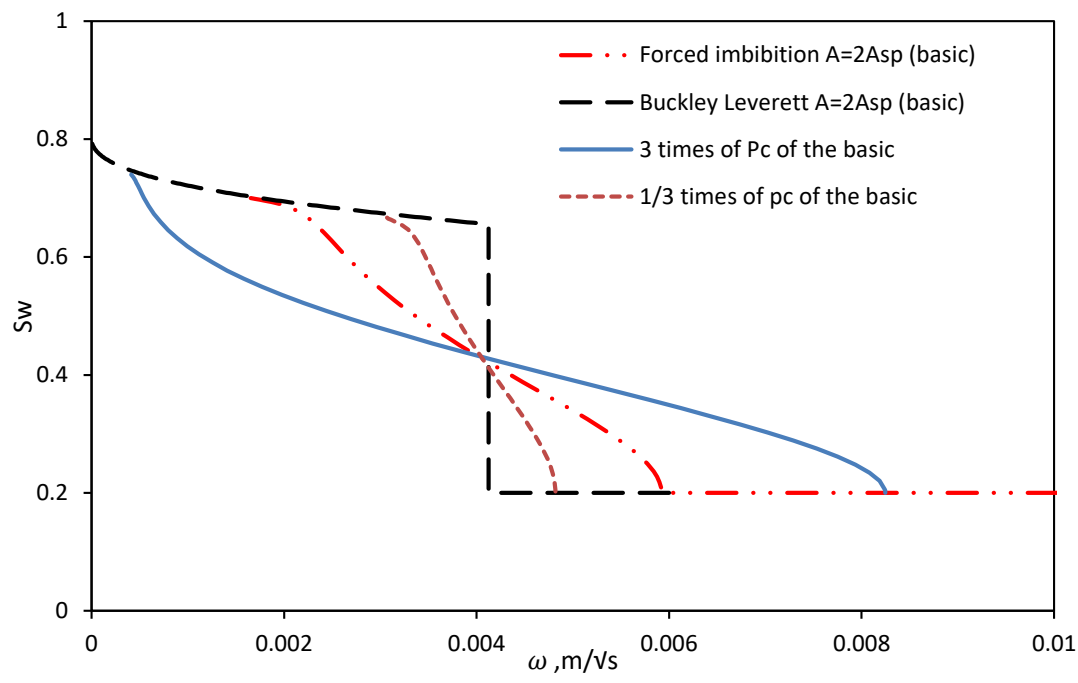

Fig. 11. Saturation propagation profiles of forced imbibition for different capillary pressure when $A=A_{s p}$. 
$x / \sqrt{t}$. The distance of the propagation is proportional to the injection velocity for both Buckley Leverett solution and our proposed solution of forced imbibition. The capillary effect disperses the sharp front of water saturation. As the injection velocity (value of $A$ ) increases, the difference between them decreases and the upper part of the saturation profile coincides with the Buckley Leverett solution. The capillary pressure effect is alleviated at a higher velocity. In this case study, when $A=5 A_{s p}$, the difference between Buckley Leverett solution and the forced imbibition is just near the frontal water saturation. Supposing this conclusion is general, for example, in some low permeability oil reservoirs, the increase of water injection rate (probably no less than 5 times of the spontaneous rate is better) will relieve the capillary effect and form a more Buckley Leverett like front and increase the oil recovery given the same injection pore volume. To further investigate the effect of capillary pressure magnitude on the two-phase flow characteristic in the reservoirs, using $A=2 A_{s p}$ as the basic case, we multiply the capillary pressure curves by 3 and $1 / 3$, the corresponding water phase profiles are shown in Fig. 11. The result is also compatible with the conclusion drawn in Fig. 10. Higher capillary pressure tends to smooth the sharp front of Buckley Leverett solution.

\section{Summary and conclusions}

A self-similar analytical solution of spontaneous and forced imbibition is proposed in this work and the corresponding concise algorithms (using a series of Runge-Kutta and Bisection methods) for solving the analytical solution are presented. This work is essential supplementary to the Buckley Leverett theory. The solution is applicable to general two-phase flow conditions. The case study validates the solution and the algorithms for both spontaneous and forced imbibition processes. The capillary effect disperses the sharp front of water saturation while a higher injection velocity can alleviate this effect and restore the solution from Buckley Leverett theory. Besides, this proposed analytical solution can be extended for other similar nonlinear partial differential equations like the form of $\frac{\partial s}{\partial t}+\frac{\partial}{\partial x}\left[g(s) \frac{\partial s}{\partial x}=0\right]$, where $s$ is the variable and $g(s)$ is a general expression of $s$ with several constants.

\section{Nomenclatures}

$A_{s p}=$ Coefficient for cocurrent spontaneous imbibition

$S_{w}^{*}=$ Maximum water saturation

$S_{w c}=$ Connate water saturation

$S_{w f}=$ Frontal water saturation

$f_{w}=$ Fractional flow function

$k_{r}=$ Relative permeability

$p_{c}=$ Capillary pressure

$A=$ Coefficient for injection velocity

$B=$ Coefficient for total velocity

$F=$ Capillary fractional flow function

$J=$ Dimensionless capillary pressure

$R F=$ Recovery factor

$S=$ Phase saturation

$a=$ Coefficient for capillary pressure $b=$ Coefficient for relative permeability curve

$c=$ Coefficient for relative permeability curve

$k=$ Permeability

$p=$ Pressure

$t=$ Time

$u=$ Darcy velocity

$x=\mathrm{X}$ direction

$\mu=$ Viscosity

$\omega=$ Transformation variable

$\phi=$ Porosity

\section{Subscripts}

$w=$ Water phase

$o=$ Oil phase

$t=$ Total phases

\section{Superscripts}

$'$ = First order derivative

" = Second order derivative

$\max =$ Maximum value

\section{Acknowledgments}

Many thanks are extended to the reviewers for giving valuable comments and improving this manuscript. The thanks also go to the Editor in Chief for the professional work of formatting and proofing this paper.

Open Access This article is distributed under the terms and conditions of the Creative Commons Attribution (CC BY-NC-ND) license, which permits unrestricted use, distribution, and reproduction in any medium, provided the original work is properly cited.

\section{References}

Bjørnarå, T.I., Mathias, S.A. A pseudospectral approach to the McWhorter and Sunada equation for two-phase flow in porous media with capillary pressure. Comput. Geosci. 2013, 17(6): 889-897.

Buckley, S.E., Leverett, M.C. Mechanism of fluid displacement in sands. Trans. AIME 1942, 146(1): 107-116.

Cai, J., Perfect, E., Cheng, C., et al. Generalized modeling of spontaneous imbibition based on Hagen-Poiseuille flow in tortuous capillaries with variably shaped apertures. Langmuir 2014, 30(18): 5142-5151.

Cai, J., Yu, B. A discussion of the effect of tortuosity on the capillary imbibition in porous media. Transp. Porous Media 2011, 89(2): 251-263.

Chapra, S., Canale, R.P. Numerical Methods for Engineers (Vol. 2). New York, USA, McGraw-Hill, 1988.

Chen, Z., Bodvarsson, G.S., Witherspoon, P.A. An Integral equation formulation for two-phase flow and other nonlinear flow problems through porous media. Paper SPE 20517 Presented at SPE Annual Technical Conference and Exhibition, New Orleans, LA, 23-16 September, 1990.

Dake, L.P. Developments in Petroleum Science. Amsterdam, Netherlands, Elsevier Science, 1978. 
Fayers, F.J., Sheldon, J.W. The effect of capillary pressure and gravity on two-phase fluid flow in a porous medium. J. Pet. Technol. 1959, 216: 147-155.

Handy, L.L. Determination of effective capillary pressures for porous media from imbibition data. Trans. AIME 1960, 219: $75-80$.

Li, C., Shen, Y., Ge, H., et al. Analysis of spontaneous imbibition in fractal tree-like network system. Fractals 2016, 24(3): 1650035.

Li, K., Horne, R.N. Characterization of spontaneous water imbibition into gas-saturated rocks. Paper SPE 62552 Presented at SPE/AAPG Western Regional Meeting, Long Beach, California, 19-22 June, 2000.

Lucas, R. Rate of capillary ascension of liquids. Kolloid Z. 1981, 23(15): 15-22.

McWhorter, D.B., Sunada, D.K. Exact integral solutions for two-phase flow. Water Resour. Res. 1990, 26(3): 399-413.

Meng, Q., Liu, H., Wang, J. A critical review on fundamental mechanisms of spontaneous imbibition and the impact of boundary condition, fluid viscosity and wettability. Adv. Geo-Energy Res. 2017, 1(1): 1-17.

Peters, E.J. Advanced petrophysics: Dispersion, interfacial phenomena (Vol. 2). Greenleaf Book Group, 2012.

Schmid, K.S., Alyafei, N., Geiger, S., et al. Analytical solutions for spontaneous imbibition: Fractional-flow theory and experimental analysis. SPE J. 2016, 21(6):
2308-2316.

Schmid, K.S., Geiger, S., Sorbie, K.S. Semianalytical solutions for cocurrent and countercurrent imbibition and dispersion of solutes in immiscible twophase flow. Water Resour. Res. 2011, 47(2): 2144-2150.

Schmid, K.S., Geiger, S. Universal scaling of spontaneous imbibition for arbitrary petrophysical properties: Waterwet and mixed-wet states and Handy's conjecture. J. Pet. Sci. Eng. 2013, 101: 44-61.

Shampine, L.F., Reichelt, M.W. The matlab ode suite. SIAM J. Sci. Comput. 1997, 18(1): 1-22.

Shen, Y., Ge, H., Zhang, X., et al. Impact of fracturing liquid absorption on the production and water-block unlocking for shale gas reservoir. Adv. Geo-Energy Res. 2018, 2(2): 163-172.

Washburn, E.W. The yynamics of capillary flow. Phys. Rev. Ser. 1921, 17(3): 273-283.

Xiao, J., Cai J., Xu, J. Saturated imbibition under the influence of gravity and geometry. J. Colloid Interface Sci. 2018, 521: 226-231.

Yokoyama, Y., Lake, L. The effects of capillary pressure on immiscible displacements in stratified porous media. Paper SPE 10109 Presented at the SPE Annual Technical Conference and Exhibition, San Antonio, USA, 4-7 October, 1981. 